\title{
Effectiveness of an Educational Program to Enhance Self-care Skills After Acute Coronary Syndrome: A Quasi-Experimental Study
}

\author{
Lisa Gomes $^{1(\bowtie)}(\mathbb{D})$ and Gorete Reis ${ }^{2}$ \\ ${ }^{1}$ Minho University, Campus de Gualtar, 4710-057 Braga, Portugal \\ lgomes@ese.uminho.pt \\ 2 Évora University, Largo do Senhor da Pobreza, 7000-811 Évora, Portugal
}

\begin{abstract}
Background: The aim of this study is to determine the effectiveness of an educational program to enhance self-care skills in patients after an acute coronary syndrome

Methods: A quasi-experimental pretest-posttest design was used in the study. A rehabilitation nurse provided an educational program (PEpSCA-CARE) to the intervention group $(n=32)$, and the control group $(n=35)$ received the conventional nursing pre-discharge care. The data was collected using the Therapeutic Self-Care Scale (TSCS) in four dimensions: medications, symptoms, activities of daily living and health status management, applied before hospital discharge and one month after hospital discharge to both groups. Patients were recruited from an intensive cardiovascular care unit during 2016.

Results: The results showed statistically significant differences between both groups $(\mathrm{p}<0.001)$. The intervention group tended to improve their self-care skills while the control group had opposite trends, self-care skills decreased.

Conclusions: According to the findings of the study, a systematized and structured educational program, is effective in developing self-care skills in patients after an acute coronary syndrome.
\end{abstract}

Keywords: Self-care skills $\cdot$ Educational program $\cdot$ Acute coronary syndrome

\section{Background}

Over the years in Portugal declining mortality and birth rates, increasing life expectancy and modifying health/disease patterns, explain the high prevalence of chronic diseases and the increasing levels of functional disability among the population. Chronic disease management requires a level of demand for permanent care and has a priority dimension in healthcare [1].

Cardiovascular Diseases are chronic diseases with the highest morbidity and mortality rate worldwide [2]. The high number of hospital admissions for Acute Coronary Syndrome and the low adherence to the Cardiac Rehabilitation (CR) constitute an opportunity for nurses to develop interventions aimed to encourage patients to take an active role in their own health care. Self-care is viewed as a process underlying 
the performance of health-related activities. The process involves the recognition of changes in a health condition that require remediation and the selection of an engagement in activities to address these changes [3].

Acute Coronary Syndromes (ACS) represents a life-threatening manifestation of atherosclerosis. It is usually caused by acute thrombosis induced by a ruptured or eroded atherosclerotic coronary plaque, with or without concomitant vasoconstriction, causing a rapid and critical reduction in blood flow [4].

ACS have been described as an extremely traumatic cardiac event and the consequences influence physical and psychosocial well-being over a significant period of time [5]. Considering that this is a chronic disease and therefore will accompany the patient throughout life, in recent year's research studies seek to understand the implications that the diagnosis, progression, and consequences of the disease have on patients and family members.

National and international associations, such as the American Association of Cardiovascular and Pulmonary Rehabilitation [6] as well as the European Association of Cardiovascular Prevention and Rehabilitation [7], recommend CR as a secondary prevention strategy. The World Health Organization defines CR as the "sum of activities required to influence favorably the underlying cause of the disease, as well as to provide the best possible physical, mental and social conditions, so that the patients may, by their own efforts, preserve or resume when lost, as normal a place as possible in the community" [8]. CR aims to reduce mortality, morbidity, and disability promotes healthy behaviors and improves quality of life. Several studies also show that CR reduces the number of hospitalizations and consequently reduces costs for the Health System [9, 10].

Despite these recommendations, the number of patients participating in cardiac rehabilitation programs is extremely low, only one-third of coronary patients have access this programs in Europe [11]. In Portugal, according to the National Coordination for Cardiovascular Diseases, the main reasons for CR underutilization are economic (financial difficulties), organizational (low medical referral rate), as well as lack of dissemination, patient's motivation, few CR clinics and poor geographical distribution of this clinics [12].

Considering that the 30 days following hospital discharge after a cardiac event is crucial for the ongoing health of ACS patients because of the high levels of stress, rehospitalization $(18.5 \%)$ and mortality $(12.5 \%)$ and it is a critical psychological period in accepting the need for lifestyle changes [13], nurse interventions during hospitalization should include promoting self-care and improving quality of life through nonpharmacological recommendations, evaluation of the signs/symptoms, stimulation of healthy habits/lifestyle, and education about the disease and the use of medicines [14].

The importance of the therapeutic regimen to control coronary disease and the consequent risks of non-adherence, make it necessary the development of strategies that promote a greater adherence to the prescribed treatment. These facts determine the importance of educational programs that enable the development of self-care skills, taking into consideration that the educational process is gradual, systematized, personalized and complemented by professionals. This requires that the patients have access to information and understand the importance of modifying their lifestyle to increase daily function. 
To study the above-mentioned issues, we provided an educational program to patients following an ACS. Therefore, we sought to test the following hypothesis: patients that attended an educational program as a nurse intervention develop self-care skills compared to patients that did not attend the program.

\section{Methods}

\subsection{Study Design}

The effectiveness of an educational program to enhance self-care skills in patients after an acute coronary syndrome for up to 1 month after hospital discharge was explored. The study used a nonequivalent, control group, pretest-posttest design due to the impossibility of group randomization. It is not possible to guarantee other common characteristics like the age and sex because of the unpredictability of acute illness episodes. Also, considering the organization of the ICCU, the participants in the intervention group were in the same ward as the non-participants, that is, if randomization was the choice, the participants of the control group would also attend the educational intervention.

\subsection{Study Participants}

The participants were adult/elderly patients diagnosed with ACS (acute myocardial infarction/unstable angina pectoris), hospitalized at an ICCU in the northern region of Portugal, speak and understand Portuguese, had preserved cognitive and verbal ability and agreed to participate in the study.

Throughout 2016, during 1-week period participants were included in the intervention group and the following week the participants were included in the control group and so on until reaching the required number of participants for our study. In the first phase, data collection was performed in the hospital, 1 month after discharge all participants were contacted by telephone.

\subsection{Educational Program (PepSCA-CARE)}

The educational process is necessary for the acquisition of knowledge and development of self-care skills. The objective of this educational program is to aware patients of the disease, the importance of changing lifestyles and controlling modifiable risk factors so they post-ACS patients (PEpSCA-CARE) was created to promote the development of self-care skills. It is intended that the actions to be implemented in this context contribute to a greater participation in the social, economic and cultural life of the communities and to guarantee a better quality of life.

In order to obtain meaningful learning for the development of skills, the implementation of the program was carried out through educational sessions built in a structured and systematic method. The training plan involved three educational areas, considering that at the end of the educational program the patient would be able to: (i) know the risks of heart disease (cognitive area); (ii) understanding that by modifying 
their behavior, their quality of life increases (affective area) and, in the psychomotor area (iii) knowing how to control the signs and symptoms of heart disease and knowing how to perform their most instrumental self-care $[15,16]$.

The PePSCA-CARE consists of 4 educational sessions, with the following pedagogical strategies: (i) information and transmission of knowledge about the situation through interviews with the nurse rehabilitation specialist; (ii) transmission of specific knowledge through the use of an educational video; (iii) verification of the information retained and clarification of doubts, using a checklist/pamphlet and (iv) verification of the knowledge retained and information about self-care performance, using telephone for follow-up interview.

The first session is individual and performed at the Intensive Coronary Care Unit (ICCU) 12 to $24 \mathrm{~h}$ after hospitalization and depending on the hemodynamic stability of the patient. An interview was conducted by the nurse rehabilitation specialist and as a didactic resource, we opted for the individual visualization of a video with the following themes and contents: How does the heart function? What is coronary heart disease? Diagnosis of coronary disease; Therapeutic Management; Modifiable Risk Factors and Healthy Lifestyle.

The second session was a group and held the following day, and aims to identify the patient's knowledge regarding the content exposed in the video and clarification of doubts. We chose to use this strategy since the beliefs about the disease as well as the personal experience also stem from the social experience [17]. Loring and Holman, explain that social persuasion can improve the perception of self-efficacy [18].

During its accomplishment, it fosters the sharing of doubts, ideas, and experiences. The third session was held on the day of hospital discharge and a checklist with the contents of the video was applied. Considering that the checklist has the following purposes: (i) organize and systematize the information so that the nurse can identify difficulties and (ii) serve as a leaflet for patient guidance when the patient has doubts at home.

The fourth and last session is performed, one month after discharge and aims to monitor the patient and reinforce the information.

Due to the geographical dispersion of the area of affluence of the hospital, this session is carried out by telephone. Telephone monitoring is seen as a good way of exchanging information, providing health education and counseling, recognizing complications, ensuring peace of mind, and providing quality post-treatment services. During this monitoring, nurses can detect abnormal conditions and implement effective actions to prevent complications.

\section{Measurement}

The Therapeutic Self-Care Scale (TSCS), was developed to assess self-care ability in acute-care settings. This scale assesses a patient's ability to perform four dimensions of self-care activities: taking medication as prescribed by the physician; identify and manage symptoms; to carry out activities of daily living and manage changes in health status [19]. The maximum score is 60 points and corresponds to a high level of performance in therapeutic self-care. 
The original version of the instrument by Doran et al. was translated, validated and adapted to the Portuguese population by Cardoso et al. [19] and shows very good internal consistency (Cronbach's $\alpha=0.979$ ), relative to the internal consistency of the original study (Cronbach's $\alpha=0.93$ ). In the current study, Cronbach's $\alpha=0.94$ at the first moment and Cronbach's $\alpha=0.96$ in the second moment.

\subsection{Data Collection Procedure}

Initial data for the pretest were collected between May and November 2016. The elements of both groups provided written consent for study participation. The cognitive status of the patients was evaluated through the Mini-Mental Status Examination and those with cognitive deficit were excluded from the program. Participants from the intervention group started the educational program 12 to $24 \mathrm{~h}$ after hospitalization. The rehabilitation nurse starts the educational program explaining what is going to happen, and the patient begins the visualization of the educational video in the ICCU. Each patient had a television so they could stop watching whenever they felt tired or could repeat if something wasn't so clear.

The study had 2 distinct moments that correspond to the admission in the ICCU pre-intervention educational evaluation and one month after hospital discharge from post-intervention evaluation.

Patients from both groups, who agreed to join the study, were advised that they would receive a telephone follow-up from a nurse one month after hospital discharge.

\subsection{Data Analysis}

For data treatment, we used descriptive and inferential statistical techniques. Statistical analysis was performed using the Statistical Package for Social Science (SPSS) software, version 23 of 2016.

The statistical techniques applied were frequency (absolute and relative), measures of central tendency (arithmetic mean and median), measures of dispersion or variability (minimum value, maximum value and standard deviation), coefficients (Cronbach's and Spearman's alpha) and tests (Chi-square test, Fisher's exact test, Mann-Whitney U test, Wilcoxon test, Spearman's correlation coefficient significance test and ShapiroWilk test as a normality test).

For all tests, the value of 0.05 was set as the limit of significance, that is, the null hypothesis was rejected when the probability of type I error (probability of rejection of the null hypothesis when it was true) was lower than the set value, when $\mathrm{p}<0.05$, that is, $\mathrm{p}<5 \%$.

\section{Results}

The results show that most of the elements of both groups had a diagnosis of acute myocardial infarction, with percentages of $90.6 \%$ and $88.6 \%$, respectively in the intervention and control groups. 
All subjects from both groups underwent invasive medical procedures, and in the majority of cases $(78.1 \%$ and $85.7 \%)$, a percutaneous coronary intervention was performed.

Regarding therapeutic self-care skills, the results allow us to verify that in the first moment of evaluation the participants of the intervention group obtained values between 54 and 60 points, with an average result of $58.31 \pm 1.20$ points. Half of the members of this group had values equal to or greater than 58.00 points and the frequency distribution was significantly different from a normal distribution $(\mathrm{p}<0.001)$.

Comparing the two groups, using the Mann-Whitney U test, we concluded that there are statistically significant differences $(\mathrm{p}<0.001)$ and the values of the central tendency measures allow us to affirm that at the first moment of evaluation, the elements of the experimental group showed a better capacity for therapeutic self-care than those in the control group.

In the second moment, all the elements of the experimental group obtained results equal to 60.00 points (maximum value of the scale of evaluation of the therapeutic selfcare). Consequently, the mean value was $60.00 \pm 0.00$ points, the median presented the same value and the frequency distribution could not be considered normal $(\mathrm{p}=0.000)$. In the control group, the results were between 21 and 58 points, with the mean value being $38.77 \pm 7.74$ points. It was verified that half of the elements of this group presented values equal or superior to 39.00 points and the distribution of frequencies reveals characteristics similar to those of a normal distribution $(p=0.883)$. The Mann-Whitney $U$ test revealed significant differences between the two groups $(\mathrm{p}<0.001)$ and comparison of the values of the central tendency measures revealed that the participants in the intervention group showed better therapeutic self-care than those in the control group.

Using the Wilcoxon test in order to compare the two groups between each evaluation moment, we verified statistically significant differences in both cases $(\mathrm{p}<0.001)$ and the comparison of the mean and median values suggests that, between the first and the second evaluation, the elements of the intervention group tended to improve their therapeutic self-care skills while in the control group the tendency was opposite, that is, self-care skills decreased (Table 1).

Table 1. Therapeutic self-care results

\begin{tabular}{l|l|l|l}
\hline Group & Intervention & Control & $\mathrm{p}$ \\
\cline { 1 - 2 } Variable & & & \\
\hline Therapeutic self-care (1st moment) & 58.31 & 43.54 & $<0.001$ \\
$\overline{\mathrm{x}}$ & 58.00 & 43.00 & \\
$\mathrm{Md}$ & 1.20 & 6.57 & \\
$\mathrm{~s}$ & 54 & 31 & \\
$\mathrm{x}_{\min }$ & 60 & 60 & \\
$\mathrm{x}_{\max }$ & $<0.001$ & 0.299 & \\
$\mathrm{p}$ & & \multicolumn{3}{|c}{ (continued) } \\
\hline
\end{tabular}


Table 1. (continued)

\begin{tabular}{l|l|l|l}
\hline Group & Intervention & Control & $\mathrm{p}$ \\
\hline Variable & & & \\
\hline Therapeutic self-care (2nd moment) & 60.00 & 38.77 & $<0.001$ \\
$\overline{\mathrm{x}}$ & 60.00 & 39.00 & \\
$\mathrm{Md}$ & 0.00 & 7.74 & \\
$\mathrm{~s}$ & 60 & 21 & \\
$\mathrm{x}_{\min }$ & 60 & 58 & \\
$\mathrm{x}_{\max }$ & 0.000 & 0.883 & \\
$\mathrm{p}$ & & & \\
$\mathbf{p}$ & $<0.001$ & $<0.001$ & \\
\hline
\end{tabular}

During hospitalization, it is possible to provide the patient with the basic knowledge so they can have the opportunity to make their own decisions regarding health care and to achieve physical condition that allows independence and autonomy once at home [20].

\subsection{Hypothesis Testing}

The data in this study corroborates that CR is associated with improvement of health status perception within cardiac patients, reinforcing their self-confidence and allowing a more complete family, social and professional reintegration, [21]. Fernandes [22] study also indicates that patients with more knowledge about the disease after the intervention, continue to gain after two months, resulting in a greater benefit from the intervention in terms of anxiety, decreased perception symptoms, concern about the disease, and the importance of emotional response. It adds that health professionals who promote educational interventions should pay particular attention to promoting knowledge about the disease and treatment.

These behavioral changes are addressed through educational interventions aimed at the patient and family/caregivers through educational sessions with information, clarification, and discussion of secondary prevention issues and accompanied by the didactic material such as pamphlets and audiovisual media [16]. In the present study, we emphasize the importance of using the didactic material because the data obtained regarding the patient's perception about the most important issue they learned at the hospital, the participants of the intervention group referred that it was the contents exposed in the video.

\section{Discussion}

CR should start at the hospital and preferably still will patient is in the ICCU [23]. At this stage, the patient is particularly available to change behaviors and understands the need to change his behaviors and adhere to a complex therapeutic regimen. During hospitalization, it is possible to provide the patient with the basic self-care knowledge to allow independence and autonomy that is required after hospital discharge [20]. 
Many of the participants of both groups, $78.1 \%$ intervention group, and $91.4 \%$ control group, had no medical indication to attend CR after discharge. Peters and Keeley's study [24] suggest advanced age, female gender, comorbidities such as heart failure, diabetes mellitus or stroke, low socioeconomic status, the absence of health insurance, or subsystem of medical assistance and distance/accessibility between home and the rehabilitation center, predictors of non-referral. Although this study does not identify the causes of non-referral to attend a CR, we emphasize that these results reinforce the importance of Phase I CR implementation in all ICCU and Cardiology Care Units and associating them with an educational nurse intervention program. It is a rethinking of the path to be taken to include the largest number of patients and to develop and test strategies to improve the overall rehabilitation of the cardiac patient. However, if the first step is not given in the hospital context, all other strategies for developing self-care skills and modeling adherence to therapeutic regimens become difficult to implement.

The study by Kadda et al. [25] about nursing education after a cardiac event, concluded that many studies have highlighted the value of nursing support in CR. Nurses and educational programs are associated with a reduction in complications rate, a decrease in anxiety levels and rehospitalization. In addition, nursing interventions aimed at adhering to healthy lifestyles effectively influence cardiac risk factors and can improve patient's prognosis. Although the results support the benefits of educational interventions, through increased knowledge and behavior change, another systematic review of the literature, warns of the need for studies on educational interventions more explicitly characterized in order to be reproduced and evaluated [26].

Often patients fear complications and rehospitalizations because of self-imposed limitation of their daily living activities. Consequently, there is dependence on personal care and daily tasks as well as emotional lability associated with sadness and depression which can lead to family and social isolation. As a consequence, there is a progressive reduction of functional capacity and quality of life that requires a multidisciplinary approach to complete rehabilitation [27].

Dickson et al. [28] explain that for better self-care management patients need vigilance and action. In our study, $65.6 \%$ of participants of the intervention group said they did not have any health professional to provide assistance and $88.6 \%$ was the percentage of response within the participants of the control group responded. These data also support the need for nurse vigilance so patients can maintain developing selfcare skills for better adherence to treatment plan and self-care management such as the ability to recognize and respond appropriately to the presence of signs and symptoms. Also in our study, most of the participants in the intervention group (68.8\%) said they had a cardiology consultation since discharged, $65.7 \%$ of the participants of the control group reply that they did not have a cardiology consultation. Patients need follow-up for an encouragement to continue in developing self-care skills and maintain a healthy lifestyle and adherence to the therapeutic regimen [23]. 


\section{Conclusions}

The results of this study indicate that the implementation of PEpSCA-CARE in ICCU can improve patient rehabilitation. Although only valid for this specific context, the results show that an educational program as a nurse intervention can make a significant difference in the patient's self-care skills. The educational program contributes to addressing unmet self-care needs, especially for patients that do not have access to CR after discharge. The overall goal of any educational program designed to promote healthcare is based on the prevention of complications and in the improvement of the quality of life [29].

Interventions aimed at developing self-care skills were fundamental so that the patients were able to manage their therapeutic regimen and adapt to their new health condition. However, there is a need for greater awareness and investment in educational nurse interventions and new online tools to facilitate an interactive communication between nurse and patient after hospital discharge so that the care provided is of an effective quality.

\section{References}

1. Grady, P.A., Gough, L.L.: Self-management: a comprehensive approach to management of chronic conditions. Am. J. Publ. Health 104(8), e25-e31 (2014). https://doi.org/10.2105/ AJPH.2014.302041

2. WHO: Noncommunicable Diseases Progress Monitor, 2017. World Health Organization, Geneva. Licence: CC BY-NC-SA 3.0 IGO (2017)

3. Sidani, S.: Self-care. In: Doran, D.M. (ed.) Nursing Outcomes: The State of the Science, 2nd edn, pp. 79-130. Jones \& Bartlett, Sudbury (2011)

4. Drakopoulou, M., Toutouzas, K., Tousoulis, D.: Chapter 2.5 - Acute coronary syndromes. In: Tousoulis, D. (ed.) Coronary Artery Disease, pp. 201-233. Academic Press (2018)

5. Chauvet-Gelinier, J.-C., Bonin, B.: Stress, anxiety and depression in heart disease patients: a major challenge for cardiac rehabilitation. Ann. Phys. Rehabil. Med. 60(1), 6-12 (2017). https://doi.org/10.1016/j.rehab.2016.09.002

6. Balady, G.J., et al.: Referral, enrollment, and delivery of cardiac rehabilitation/secondary prevention programs at clinical centers and beyond: a presidential advisory from the American Heart Association. Circulation 124(25), 2951-2960 (2011). https://doi.org/10. 1161/CIR.0b013e31823b21e2

7. Piepoli, M.F., et al.: 2016 European Guidelines on cardiovascular disease prevention in clinical practice: the sixth joint task force of the European Society of cardiology and other societies on cardiovascular disease prevention in clinical practice (constituted by representatives of 10 societies and by invited experts) developed with the special contribution of the European Association for Cardiovascular Prevention \& Rehabilitation (EACPR). Eur. Heart J. 37(29), 2315-2381 (2016). https://doi.org/10.1093/eurheartj/ehw106

8. World Health Organization: Needs and Action Priorities in Cardiac Rehabilitation and Secondary Prevention in Patients with Coronary Heart Disease. WHO Regional Office for Europe, Geneva (1993) 
9. Magalhães, S., et al.: Efeitos a longo prazo de um programa de reabilitação cardíaca no controlo dos fatores de risco cardiovasculares. Rev. Port. Cardiol. 32(3), 191-199 (2013). https://doi.org/10.1016/j.repc.2012.08.005

10. Foster, J.G., Lewis, S.F., Hennekens, C.H.: Editorial commentary: cardiac rehabilitation: major benefits and minor risks. Trends Cardiovasc. Med. 27(6), 426-427 (2017). https://doi. org/10.1016/j.tcm.2017.03.001

11. Bjarnason-Wehrens, B., et al.: Cardiac rehabilitation in Europe: results from the European cardiac rehabilitation inventory survey. Eur. J. Cardiovasc. Prev. Rehabil. 17(4), 410-418 (2010). https://doi.org/10.1097/HJR.0b013e328334f42d

12. Coordenação Nacional para as Doenças Cardiovasculares, 2009. Reabilitação Cardíaca: Realidade Nacional e Recomendações Clínicas. http://www2.portaldasaude.pt/NR/rdonlyres/ 466A7B26-7BB4-48D9-9DC1-FBAE234AA579/0/ReabilitacaoCardiaca.pdf

13. Chen, H.Y., et al.: Decade-long trends in 30-day rehospitalization rates after acute myocardial infarction. J. Am. Heart Assoc. 4(11), e002291 (2015). https://doi.org/10.1161/ JAHA.115.002291

14. Boisvert, S., Proulx-Belhumeur, A., Gonçalves, N., Doré, M., Francoeur, J., Gallani, M.C.: An integrative literature review on nursing interventions aimed at increasing self-care among heart failure patients. Revista Latino-Americana de Enfermagem 23(4), 753-768 (2015). https://doi.org/10.1590/0104-1169.0370.2612

15. Adams, N.E.: Bloom's taxonomy of cognitive learning objectives. J. Med. Libr. Assoc. JMLA 103(3), 152-153 (2015). https://doi.org/10.3163/1536-5050.103.3.010

16. Rice, H., Say, R., Betihavas, V.: The effect of nurse-led education on hospitalisation, readmission, quality of life and cost in adults with heart failure: a systematic review. Patient Educ. Couns. 101(3), 363-374 (2018). https://doi.org/10.1016/j.pec.2017.10.002

17. Sousa, M.R., Martins, T., Pereira, F.: O refletir das práticas dos enfermeiros na abordagem à pessoa com doença crónica. Revista de Enfermagem Referência 6, 55-63 (2015)

18. Holman, H., Lorig, K.: Patient self-management: a key to effectiveness and efficiency in care of chronic disease. Public Health Rep. 119(3), 239-243 (2004). https://doi.org/10.1016/j.phr. 2004.04.002

19. Cardoso, A., Queiros, P., Ribeiro, C., Amaral, A.: Cultural adaptation and psychometric properties of the portuguese version of the therapeutic self-care scale. Int. J. Caring Sci. 7(2), 426 (2014)

20. Mampuya, W.M.: Cardiac rehabilitation past, present and future: an overview. Cardiovasc. Diagn. Ther. 2(1), 38-49 (2012). https://doi.org/10.3978/j.issn.2223-3652.2012.01.02

21. Rocha, E., Nogueira, P.: As doenças cardiovasculares em Portugal e na região Mediterrânica: uma perspetiva epidemiológic. Revista Factores de Risco 36, 35-44 (2015)

22. Fernandes, A.: Avaliação da eficácia de um programa de intervenção psicológica breve em pacientes pós-síndrome coronária aguda. (PhD), Universidade do Minho, Braga (2012)

23. Abreu, A., et al.: Mandatory criteria for cardiac rehabilitation programs: 2018 guidelines from the Portuguese society of cardiology. Rev. Port. Cardiol. 37(5), 363-373 (2018). https://doi.org/10.1016/j.repc.2018.02.006

24. Peters, A.E., Keeley, E.C.: Trends and predictors of participation in cardiac rehabilitation following acute myocardial infarction: data from the behavioral risk factor surveillance system. J. Am. Heart Assoc. 7(1) (2017). https://doi.org/10.1161/jaha.117.007664

25. Kadda, O., Marvaki, C., Panagiotakos, D.: The role of nursing education after a cardiac event. Health Sci. J. 6(4), 634 (2012)

26. de Melo Ghisi, G.L., da Silva Chaves, G.S., Britto, R.R., Oh, P.: Health literacy and coronary artery disease: a systematic review. Patient Educ. Couns. 101(2), 177-184 (2018). https://doi.org/10.1016/j.pec.2017.09.002 
27. Ades, P.A., et al.: Cardiac rehabilitation exercise and self care for chronic heart failure. JACC Heart Fail. 1(6), 540-547 (2013). https://doi.org/10.1016/j.jchf.2013.09.002

28. Dickson, V.V., Nocella, J., Yoon, H.-W., Hammer, M., Melkus, G.D., Chyun, D.: Cardiovascular disease self-care interventions. Nurs. Res. and Pract. 2013, Article ID 407608 (2013). https://doi.org/10.1155/2013/407608

29. Megari, K.: Quality of life in chronic disease patients. Health Psychol. Res. 1(3), e27 (2013). https://doi.org/10.4081/hpr.2013.e2 